\title{
Realities of Pharmacogenomic and Minimizing Misconceptions and Medication Misadventures
}

\author{
Benjamin Duong, PharmD
}

Nemours Children's Health Delaware, Precision Medicine, Clinical Pharmacogenomics Service

\begin{abstract}
Pharmacogenetics allows providers to enhance their treatment decisions for common medications used in certain conditions such as depression, gastroesophageal reflux disease (GERD), pain, and acute lymphoblastic leukemia. A precision medicine approach combines pharmacogenetics (when appropriate) with other clinical and environmental factors to minimize trial-and-error of treatment. Public awareness of the impact of pharmacogenetics on treatment decisions is growing, and healthcare should be aware of the resources supporting it. Pharmacogenetics may seem daunting, but the accessibility of pharmacogenetic testing has improved with growing availability of evidence-based clinical recommendations, pharmacogenetic tests, clinical decision support resources, insurance coverage, and digestible education materials. As precision medicine and precision public health expands over the next decade, pharmacogenetic testing will continuously grow to be cheaper and part of routine genetic or genomic screenings, and be another common test - like liver or kidney function tests - that can enhance treatment decisions.
\end{abstract}

\section{Introduction}

Pharmacogenetics (PGx) examines genetic variability as one of many contributing factors in the variability of drug exposure and response (i.e. side effects, treatment failure). ${ }^{1}$ It is one aspect of "precision medicine," an approach to improve health by accounting for individual variability in genes, environment, and lifestyle. ${ }^{2}$ Unlike rare genetic diseases, pharmacogenetic variants warranting dose adjustments are relatively common. In a study containing over 10,000 patients, over $91 \%$ will have at least one actionable variant involved in five drug-gene interactions. ${ }^{3}$

PGx examples to minimize medication misadventures in certain populations include:

- TPMT and NUDT15 genotyping in patients requiring thiopurine (used in acute lymphoblastic leukemia, ulcerative colitis, and Crohn's disease) will identify $10 \%$ of the population at risk for severe thiopurine-induced myelosuppression;

- CYP2C19 and CYP2D6 genotyping in patients with depression will identify patients at risk of adverse effects or treatment failure to their certain first-life antidepressants; and

- Panel-based PGx tests in cancer patients may guide certain supportive care medications such as CYP2D6 for opioids, CYP2D6 for antiemetics, CYP2C19 and CYP2D6 for antidepressants, and CYP2C19 for proton pump inhibitors.

The reality of PGx testing is that PGx is only one piece of the puzzle, and it should be used to enhance, not replace, evidence-based treatment decisions, along with other clinical (e.g., drugdrug interaction, condition) and environmental considerations (e.g., adherence, cost, exercise). It can help the healthcare provider narrow down treatment options or assist in predicting dosage for 
certain medications by identifying medications with increased risk of adverse effects or therapeutic failure based on their known PGx variants. There are a lot of misconceptions advertising that PGx identify the "magic bullet" that will treat the condition without side effects. However, PGx cannot predict all adverse reactions to medication, predict risk of a specific side effect for all medication, diagnose a disease, or find the perfect regimen with no risk. ${ }^{4}$

Due to widespread use of direct-to-consumer genetic testing, patients are becoming more informed of how genetics can play a role in their healthcare. They are coming to their health care providers expecting them to be able to order the right test and apply it to their care. Although just under $80 \%$ of pediatricians and primary care providers believe PGx can improve care, less than $10 \%$ of pediatrician were initially familiar with $\mathrm{PGx}^{5}$ and $26 \%$ of adult primary care providers expressed confidence in using PGx in a treatment decision. ${ }^{6}$ The purpose of this article is to briefly increase awareness of publicly available resources to utilize PGx in treatment decisions, how accessibility is improving using PGx results, and briefly highlight the implementation of a PGx service at Nemours Children's Health System.

\section{Available PGx Resources}

There are dozens of health systems that have now implemented PGx into their clinical practices across the country with evidence-based recommendations, PGx testing, and clinical decision support platforms in their electronic health records (EHR). The Implementing Genomics in Practice (IGNITE) Network is an NIH-funded network includes over 20 institutions dedicated to support genomic implementation in healthcare. ${ }^{7}$ The IGNITE Network's Genomic Medicine Knowledgebase's IGNITE Toolbox (www.gmkb.org) consists of their collection of genomic implementation resources, clinical decision support examples, and education material publicly available for other institutions to consider. This knowledge base also provides an Implementation Guide that outlines the steps and resources on implementing CYP2C19-clopidogrel and CYP2D6-opioids. The implementation science framework that was used to design the implementation guide can be applied other specific drug-gene interactions of interest or a PGx process in general. ${ }^{8}$ The initial steps identified include ensuring there is sufficient evidence, laboratory testing process, reimbursement, clinical decision support, and education.

\section{Clinical Evidence}

To apply PGx clinically, there should be significant evidence to support the drug-gene interaction. Fortunately, there are multiple established sources with a collection of evidencebased drug-gene interactions through the Food and Drug Administration (FDA), the Clinical Pharmacogenetics Implementation Consortium (CPIC), and the Pharmacogenomics Knowledge Base (PharmGKB). The FDA provides PGx information and/or recommendations through their Table of Pharmacogenetic Association, Table of Pharmacogenomic Biomarkers, and within the drug labeling. ${ }^{9,10}$ CPIC (www.cpicpgx.org) is an international consortium of clinicians and scientists that develops clinical PGx guidelines with structured interpretations and recommendations of evidence-based gene-drug interactions. The CPIC guidelines utilize a rigorous evidence evaluation process and adhere to the Institute of Medicine's Standards for Developing Trustworthy Clinical Practice Guidelines. ${ }^{11}$ As of December 2021, there are 26 published CPIC guidelines that correspond to over 80 medications spanning across multiple therapeutic areas such as psychiatry, cardiology, pain management, and hematology/oncology. Lastly, PharmGKB (www.pharmgkb.org), is a freely available public resource that users can 
search for a drug or gene, and find any existing evidence and recommendations from the FDA, CPIC, or other expert groups. ${ }^{12}$ These groups evaluate the evidence and disseminate publiclyavailable evidence-based recommendations that providers without a genetics background would be able to apply in their practice.

\section{Laboratory}

Single and/or multi-gene PGx tests can be obtained through direct-to-consumer genetic testing companies, large clinical laboratory networks with thousands of sites across the country, institutional pathology or molecular diagnostic laboratories, and commercial provider-ordered combinatorial pharmacogenomic testing companies that utilize a proprietary algorithm. Despite the large availability of PGx tests, the genetic variants, nomenclature, and gene-drug interaction recommendations may be discordant from other laboratories and CPIC's recommendation and translation. ${ }^{13}$ Unfortunately, the current challenge to PGx testing is the deviation by most PGx labs from CPIC phenotype translation and recommendations. The misleading information may lead to unnecessary avoidance of first-line agents that do not have enough evidence to have a drug-gene interaction, and increased utilization of medication that is not appropriate for the patient.

Selection of a laboratory should include four domains: pharmacogene selection, logistics, reporting of results, and test costs and reimbursement. ${ }^{14}$ The pharmacogene selected and results reported should use standardized nomenclature and recommendations as outlined in CPIC's PGx standardization publication ${ }^{15}$ and genotype-phenotype translation. The genes and variants tested should also be representative of the population and cover variants with established reference material on the variant's impact to the enzyme's functionality and multi-ethnic frequency as such by the Association of Molecular Pathology for CYP2C19 and CYP2D6. ${ }^{16,17}$ Logistics that should be considered are the type of sample collection, turnaround time for results, and documentation formed/required. Lastly, cost and reimbursement should be reasonable and covered by insurance. ${ }^{14}$ Despite possible discrepancies in the recommendations provided, providers can use the genotype results and check with PharmGKB and CPIC for available clinical recommendations.

\section{Integration/Clinical Decision Support}

PGx clinical decision support (CDS) is the integration of PGx results into the electronic medical records to prevents errors and improve health. ${ }^{18}$ Types of CDS include active CDS and passive CDS. Active CDS are typically interruptive alerts when there is a significant drug-gene interaction or when a drug is ordered and PGx testing is recommended. Passive CDS is available PGx information if the user chooses to seek it (e.g. a PGx profile listing all the possible druggene interactions or embedded PGx results within the drug's order information). CDS usually requires PGx information to be uploaded as discrete variables to enable rule-based decisions. Depending on the institution's capabilities, they may decide to create PGx CDS using internal resources or outsource with the multiple commercial PGx CDS platforms. Ideally, PGx CDS are seamlessly integrated with the electronic medical records and provide PGx recommendations concordant with CPIC or FDA recommendations. ${ }^{18,19}$ 


\section{Insurance Coverage}

Although PGx testing is typically cheaper than traditional diagnostic genetic tests, cost and lack of insurance coverage is a barrier in PGx testing adoption. PGx test cost averages about $\$ 300$ depending on the laboratory, however most health insurance companies typically deny reimbursement of PGx testing as they are considered investigational and/or experimental. This may change soon as, effective December 12, 2021, PGx testing will be covered by Medicare across 40 of the 50 states as Novitas Solutions, and First Coast Medicare Administrative Contractor (MAC) jurisdiction harmonizes with Molecular Diagnostic Services (MolDx) local coverage determination (LCD) which involves four other MACs. ${ }^{20-22}$ Novitas Solution and First Coast MACs includes Colorado, New Mexico, Oklahoma, Texas, Arkansas, Louisiana, Mississippi, Delaware, Maryland, Pennsylvania, New Jersey, District of Columbia, and Florida. This marks another milestone in the adoption and accessibility of PGx testing as tool to enhance treatment decisions and minimize risk of treatment failures or adverse effects.

Per the LCD, PGx testing is considered medically reasonable and necessary if the patient is indicated for a medication with a known gene-drug interaction; and the PGx test meets standards (as evaluated by a scientific, transparent, peer-reviewed process) and is determined to demonstrate actionability in clinical decision making (by CPIC guidelines level A or B level of evidence, FDA's Table of Pharmacogenomic Biomarkers in Drug Labeling, the Table of Pharmacogenetic Associations, or FDA drug labeling). PGx testing is not medically reasonable and necessary if the analytical validity, clinical validity, or clinical utility is not established, or the test investigates the duplicative genetic information. ${ }^{20,21}$ The language from the LCD may restrict coverage for combinatorial PGx tests that rely on proprietary algorithms and provide clinical recommendations on drug-gene interactions classified as low level of evidence by CPIC and PharmGKB. With the new coverage, commercial payers and Medicaid are expected to adopt similar coverages to harmonize with their LCD.

\section{Education}

Like with any intervention, the patient should be educated of the benefits and limitations of PGx testing. Education resources for patients should highlight what PGx can and cannot do, such as the fact that PGx testing cannot predict all adverse reactions to medication, specific side effects for all medications, or the perfect "magic pill." If a combinatorial PGx test is used, the patient should be educated that the report may contain low-evidence recommendations, and that categorized pharmacogenomic recommendations with a "Red" indication ("Significant DrugGene Interaction") may be clinically appropriate more than the "Green" list ("No Drug-Gene Interaction").

PGx education in various health professions is increasing as schools and organizations have been embedding PGx education into their curriculums and continuous education opportunities. Healthcare professionals seeking formal PGx education on how to effectively apply or implement PGx in their practice can find live or online PGx certificate programs from professional societies (e.g., American Society of Health-System Pharmacists, American College of Clinical Pharmacology) and academic institutions (e.g., University of Pittsburgh, University of Florida). 


\section{Nemours Clinical Pharmacogenomics Service}

The resources and considerations above were vital in developing the dedicated Clinical Pharmacogenomic Service at Nemours Children's Health launching in February 2020, one of only dozen of such services implemented across the country by pediatric health systems. ${ }^{23}$ This service is managed by pharmacists with clinical PGx residency training to construct PGx resources across the enterprise and assist providers with utilizing PGx with their care. The service's implementation responsibilities include developing an in-house PGx panel with the Nemours' CLIA-certified Molecular Diagnostic Laboratory, customizing CDS processes specific to the pediatric population that seamlessly incorporate PGx results into the medical record, fire interruptive drug-gene interaction best practice alerts as a last-line of defense, and patient and provider education. The Nemours Clinical Pharmacogenomics Service direct patient care includes assisting providers to determine how the PGx may be used, coordinating logistics with insurance authorization and DNA sample collection (blood or buccal swab), interpreting any PGx results regardless of the source (direct-to-consumer, commercial, secondary/incidental findings from diagnostic genetic tests, or the Nemours in-house panel), and reviewing the results with the patient and provider. Many of the patients consulted were patients with mental health conditions, about half of which heard about PGx testing from another parent, family member, or online forum.

\section{Discussion}

Accessibility to PGx has grown tremendously over the past decade with stronger evidence supporting utilization and clinical guidelines, increasing availability clinical PGx tests, growing insurance coverage for more affordable tests, and advancing clinical decision support integration. Over the next few years, PGx is expected to transition from a more reactive approach where testing is ordered by specialists and/or when patients have already failed multiple medications, to earlier in the diagnosis using a preemptive panel approach before a medication is indicated. Adoption of preemptive PGx testing will allow for a healthier population by enhancing treatment decisions and minimizing trial and error. This will contribute to one piece of the precision medicine puzzle and the emerging concept of "Precision Public Health," with the goal of providing right treatment for the right population at the right time. ${ }^{24}$ In 2030 , it is envisioned that precision medicine will have a wealth of data that includes not only genetics but also patient's environmental factors and medical records. PGx testing will become more routine and stored in "genome-aware" EHR that is standardized and automatically updated from central guidelines, and portable across various institutions. ${ }^{2}$

In conclusion, there is substantial availability of PGx testing and resources that should allow application across the country. There is still a need for harmonization between PGx laboratories with evidence-based interpretation and recommendation as well as coverage in a variety insurance companies, but recently PGx accessibility has become widespread and ready for application as one piece of the puzzle to enhance treatment decisions.

Correspondence: Dr. Duong can be emailed at benjamin.duong@nemours.org

\section{References}

1. Ramsey, L. B., Brown, J. T., Vear, S. I., Bishop, J. R., \& Van Driest, S. L. (2020, January 6). Gene-based dose optimization in children. Annual Review of Pharmacology and 
Toxicology, 60, 311-331. PubMed https://doi.org/10.1146/annurev-pharmtox-010919$\underline{023459}$

2. Denny, J. C., \& Collins, F. S. (2021, March 18). Precision medicine in 2030-seven ways to transform healthcare. Cell, 184(6), 1415-1419. PubMed https://doi.org/10.1016/j.cell.2021.01.015

3. Van Driest, S. L., Shi, Y., Bowton, E. A., Schildcrout, J. S., Peterson, J. F., Pulley, J., . . . Roden, D. M. (2014, April). Clinically actionable genotypes among 10,000 patients with preemptive pharmacogenomic testing. Clinical Pharmacology and Therapeutics, 95(4), 423-431. Epub2013Nov19. PubMed

4. Wake, D. T., Ilbawi, N., Dunnenberger, H. M., \& Hulick, P. J. (2019, November). Pharmacogenomics: Prescribing Precisely. The Medical Clinics of North America, 103(6), 977-990. PubMed https://doi.org/10.1016/j.mcna.2019.07.002

5. Rahawi, S., Naik, H., Blake, K. V., Owusu Obeng, A., Wasserman, R. M., Seki, Y., .. . Scott, S. A. (2020, May). Knowledge and attitudes on pharmacogenetics among pediatricians. Journal of Human Genetics, 65(5), 437-444. PubMed https://doi.org/10.1038/s10038-020-0723-0

6. Smith, D. M., Namvar, T., Brown, R. P., Springfield, T. B., Peshkin, B. N., Walsh, R. J., . . Swain, S. M. (2020, October). Assessment of primary care practitioners' attitudes and interest in pharmacogenomic testing. Pharmacogenomics, 21(15), 1085-1094. PubMed https://doi.org/10.2217/pgs-2020-0064

7. Sperber, N. R., Dong, O. M., Roberts, M. C., Dexter, P., Elsey, A. R., Ginsburg, G. S., . . Orlando, L. A. (2021, July 8). Strategies to integrate genomic medicine into clinical care: Evidence from the IGNITE network. Journal of Personalized Medicine, 11(7), 647. PubMed https://doi.org/10.3390/jpm11070647

8. Duong, B. Q., Arwood, M. J., Hicks, J. K., Beitelshees, A. L., Franchi, F., Houder, J. T., . . . Wiisanen, K., \& the IGNITE Network. (2020, July 17). Development of customizable implementation guides to support clinical adoption of pharmacogenomics: Experiences of the implementing genomics in practice (IGNITE) network. Pharmacogenomics and Personalized Medicine, 13, 217-226. PubMed

9. US Food and Drug Administration. (2021). Table of pharmacogenetic associations. https://www.fda.gov/medical-devices/precision-medicine/table-pharmacogeneticassociations

10. US Food and Drug Administration. (2021). Table of pharmacogenomic biomarkers. https://www.fda.gov/drugs/science-and-research-drugs/table-pharmacogenomic-biomarkersdrug-labeling

11. Relling, M. V., Klein, T. E., Gammal, R. S., Whirl-Carrillo, M., Hoffman, J. M., \& Caudle, K. E. (2020, January). The clinical pharmacogenetics implementation consortium: 10 Years Later. Clinical Pharmacology and Therapeutics, 107(1), 171-175. PubMed https://doi.org/10.1002/cpt.1651

12. Gong, L., Whirl-Carrillo, M., \& Klein, T. E. (2021). PharmGKB, an integrated resource of pharmacogenomic knowledge. Current protocols, 1(8), e226. 
13. Bousman, C. A., \& Dunlop, B. W. (2018, September). Genotype, phenotype, and medication recommendation agreement among commercial pharmacogenetic-based decision support tools. The Pharmacogenomics Journal, 18(5), 613-622. PubMed https://doi.org/10.1038/s41397-018-0027-3

14. Vo, T. T., Bell, G. C., Owusu Obeng, A., Hicks, J. K., \& Dunnenberger, H. M. (2017, September). Pharmacogenomics implementation: Considerations for selecting a reference laboratory. Pharmacotherapy, 37(9), 1014-1022. PubMed https://doi.org/10.1002/phar.1985

15. Caudle, K. E., Dunnenberger, H. M., Freimuth, R. R., Peterson, J. F., Burlison, J. D., WhirlCarrillo, M., . . . Hoffman, J. M. (2017). Standardizing terms for clinical pharmacogenetic test results: consensus terms from the Clinical Pharmacogenetics Implementation Consortium (CPIC). Genetics in medicine: official journal of the American College of Medical Genetics, 19(2), 215-223.

16. Pratt, V. M., Del Tredici, A. L., Hachad, H., Ji, Y., Kalman, L. V., Scott, S. A., \& Weck, K. E. (2018, May). Recommendations for clinical CYP2C19 genotyping allele selection: A report of the Association for Molecular Pathology. The Journal of molecular diagnostics. $J$ Mol Diagn, 20(3), 269-276. PubMed https://doi.org/10.1016/j.jmoldx.2018.01.011

17. Pratt, V. M., Cavallari, L. H., Del Tredici, A. L., Gaedigk, A., Hachad, H., Ji, Y., ... Weck, K. E. (2021, September). Recommendations for Clinical CYP2D6 Genotyping Allele Selection: A Joint Consensus Recommendation of the Association for Molecular Pathology, College of American Pathologists, Dutch Pharmacogenetics Working Group of the Royal Dutch Pharmacists Association, and the European Society for Pharmacogenomics and Personalized Therapy. J Mol Diagn, 23(9), 1047-1064. PubMed https://doi.org/10.1016/j.jmoldx.2021.05.013

18. Wake, D. T., Smith, D. M., Kazi, S., \& Dunnenberger, H. M. (2021, August 8). (2021). Pharmacogenomic clinical decision support: A review, how-to guide, and future vision. Clinical Pharmacology and Therapeutics; Advance online publication. PubMed

19. Cook, K. J., Duong, B. Q., Seligson, N. D., Arn, P., Funanage, V. L., Gripp, K. W., . . . Blake, K. V. (2021, September). Key considerations for selecting a genomic decision support platform for implementing pharmacogenomics. Clinical Pharmacology and Therapeutics, 110(3), 555-558. PubMed https://doi.org/10.1002/cpt.2328

20. Novitas Solutions, Inc. (2021) Local coverage determination (LCD): pharmacogenomics testing (L39063).

21. First Coast Service Options Solutions, Inc. (2021) Local coverage determination (LCD): pharmacogenomics testing (L39073).

22. Palmetto, G.B.A. (2020). Local coverage determination (LCD): MolDX: Pharmacogenomics Testing (L38294).

23. Brown, J. T., Ramsey, L. B., Van Driest, S. L., Aka, I., \& Colace, S. I. (2021, March). Characterizing pharmacogenetic testing among children's hospitals. Clinical and Translational Science, 14(2), 692-701. PubMed https://doi.org/10.1111/cts.12931

24. Khoury, M. J., Bowen, M. S., Clyne, M., Dotson, W. D., Gwinn, M. L., Green, R. F., \& Yu, W. (2018). From public health genomics to precision public health: a 20 -year journey. 
Doi: 10.32481/djph.2021.12.006

Genetics in medicine: official journal of the American College of Medical Genetics, 20(6), 574-582.

\section{Copyright (c) 2021 Delaware Academy of Medicine / Delaware Public Health Association.}

This is an Open Access article distributed under the terms of the Creative Commons Attribution Non-Commercial License (https://creativecommons.org/licenses/by-nc-nd/4.0/) which permits unrestricted non-commercial use, distribution, and reproduction in any medium, provided the original work is properly cited. 